Bull. Mater. Sci., Vol. 8, No. 4, October 1986, pp. 511-517.

(C) Printed in India.

\title{
Periodic crystallization of barium oxalate in silica hydrogel
}

\author{
S M DHARMA PRAKASH and P MOHAN RAO \\ Department of Physics, Mangalore University, Mangala Gangothri, Mangalore 574 199, \\ India \\ MS received 1 March 1986; revised 5 May 1986
}

\begin{abstract}
The experimental conditions for obtaining periodic crystallization of barium oxalate in silica hydrogel are investigated. The Liesegang rings formed by the reaction of oxalic acid and barium chloride are studied. The dependence of velocity constant and the spacing coefficient on $\mathrm{pH}$, concentrations of inner and outer electrolytes and temperature have also been studied and a possible mechanism for the behaviour is suggested. The deviations from Isemura's general observations are interpreted by considering the microcrystals constituting the rings and the irreversibility of inner and outer electrolytes.
\end{abstract}

Keywords. Barium oxalate; silica hydrogel; periodic crystallization.

\section{Introduction}

Periodic precipitation in the form of concentric rings was first observed by Liesegang (1896) during his experiments of chemical reactions in gels. Several theories of Liesegang ring phenomenon have been proposed viz the supersaturation theory (Ostwald 1897; Wagner 1950), the absorption theory (Bradford 1922; Dhar and Chatterji 1922; Shinohara 1970), the coagulation theory (Dhar and Chatterji 1922), the diffusion wave theory (Ostwald 1925), the comprehensive theory (Hedges 1932) and the flocculation theory (Shinohara 1970). All these theories only partially explain the experimental observations. Two laws govern Liesegang phenomenon. The first one is the spacing law given by Jablczynski (1923)

$$
X_{n}=a S^{n},
$$

where $X_{n}$ is the distance of the $n$th ring from the origin of diffusion, and $a$ and $S$ are constants, $S$ being known as the spacing coefficient. The second law is the time law of Morce and Pierce (1903),

$$
\left(X_{n} / t_{n}^{1 / 2}\right)=K
$$

where $X_{n}$ is the distance to which the precipitate penetrates in time $t_{n}$ and $K$ is the velocity constant.

The present paper reports our investigation of the conditions for obtaining periodic crystallization of barium oxalate in silica hydro gel. The effects of various factors like the temperature, the concentration of the inner and outer reactants and the $\mathrm{pH}$ of the gel medium are considered.

\section{Experimental}

Experiments were carried out in corning glass test tubes (inner diameter $2.5 \mathrm{~cm}$, length $25 \mathrm{~cm}$ ). The chemicals used were: sodium metasilicate, oxalic acid (AR) and 
$\mathrm{BaCl}_{2} \cdot 2 \mathrm{H}_{2} \mathrm{O}$ (AR). The gel was set by mixing sodium metasilicate solution of specific gravity, 1.03 with aqueous solution of $0.5 \mathrm{M}$ oxalic acid in proportions such that the $\mathrm{pH}$ of the resulting solutions lay at $4.15,4.35,4.55,4.75$ and 4.95 measured immediately after mixing. Barium chloride solutions of $0.7,0.5,0.3$ and $0.1 \mathrm{M}$ concentration were carefully poured into the tubes over the set gel. The experiments were performed at $31,37,43$ and $49^{\circ} \mathrm{C}$. Cathetometer was used for measuring the position of the rings. Well-spaced rings were nucleated after 20-30 minutes. The time of formation and distance of each ring was recorded. The constants $K$ and $S$ for individual rings and hence for each experimental tube were computed. The Liesegang rings formed in the range are displayed in figure 1.

\section{Observations and discussion}

In accordance with the time law of Jablczynski (1923), the velocity constant $K$ is independent of $X_{n}$ and $t_{n}$. Similarly the spacing co-efficient $S$ is independent of the order of the ring according to the spacing law of Morce and Pierce (1903). But it is observed that both $K$ and $S$ depend on the concentration of the inner and outer electrolytes and their diffusion coefficients.

\subsection{Variation of $K$ and $S$ with $p H$}

In figure 2, time and space constants are plotted against $\mathrm{pH}$ of the reaction medium, taking temperature and concentration of the outer electrolyte as fixed parameters. The graphs reveal that as the $\mathrm{pH}$ of the gel medium increases, the velocity constants $(K)$ and the space constants $(S)$ decrease. The points on the graph for $S$ at different temperatures overlap and show small changes in the value of $S$ with temperature.

Though the $\mathrm{pH}$ is the measure of acidity of the gel, it also indicates the concentration of inner electrolyte (or oxalate ions in the present case). Hence, for a particular concentration of the outer electrolyte both $K$ and $S$ decrease almost linearly with increase in the concentration of the inner electrolyte. Similar results have been reported from the studies of Liesegang phenomena of various systems (Gnanam et al 1979; Kanniah et al 1979; Arora et al 1982). Thus the inner electrolyte also plays an important role in the Liesegang phenomenon (Matalon and Packter 1955).

The observed decrease of $K$ can be attributed to the increased hardness of the gel which in turn decreases the diffusion rate and increases the time of ring formation. The decrease of $S$ in the present study may be explained as follows: At higher $\mathrm{pH}$, though a lesser amount of $\mathrm{Ba}^{2+}$ ions diffuse through the pores of the hardened gel, the concentration of the uniformly spread oxalate ions is quite high. So, when a ring is formed, oxalate ions in the immediate vicinity would be sufficient to complete the reaction with the incoming barium ions. The region from which the oxalate ions diffuse upwards is small and consequently the rings tend to form more closely separated in harder gels, thereby showing relatively lower values of $S$.

3.2 Variation in space and time constants with the concentration of the outer electrolyte

Figure 3 depicts the variation of $K$ and $S$ with the change in the concentration of feed solution at different $\mathrm{pH}$ of the reaction medium and different temperatures. 


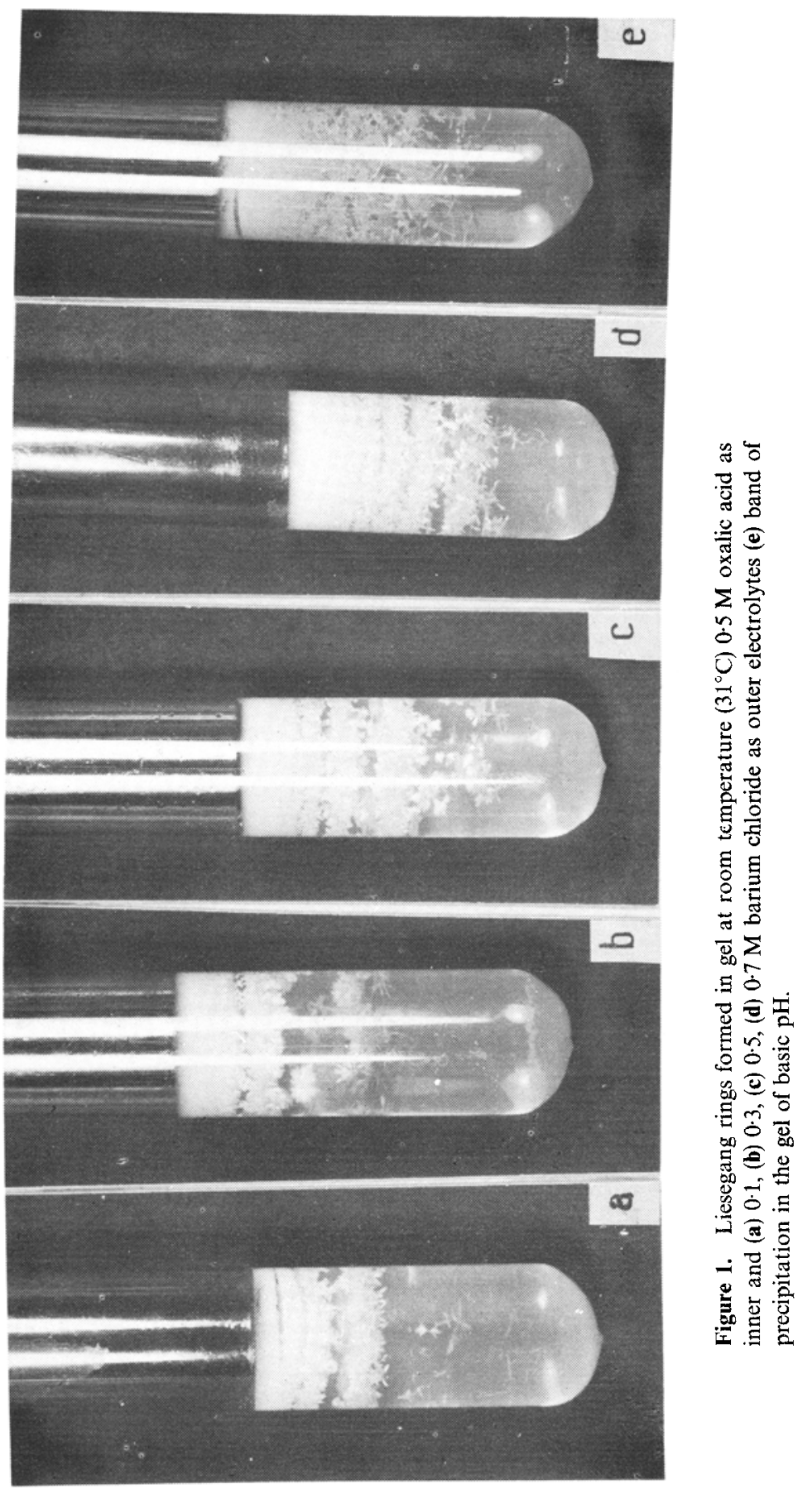



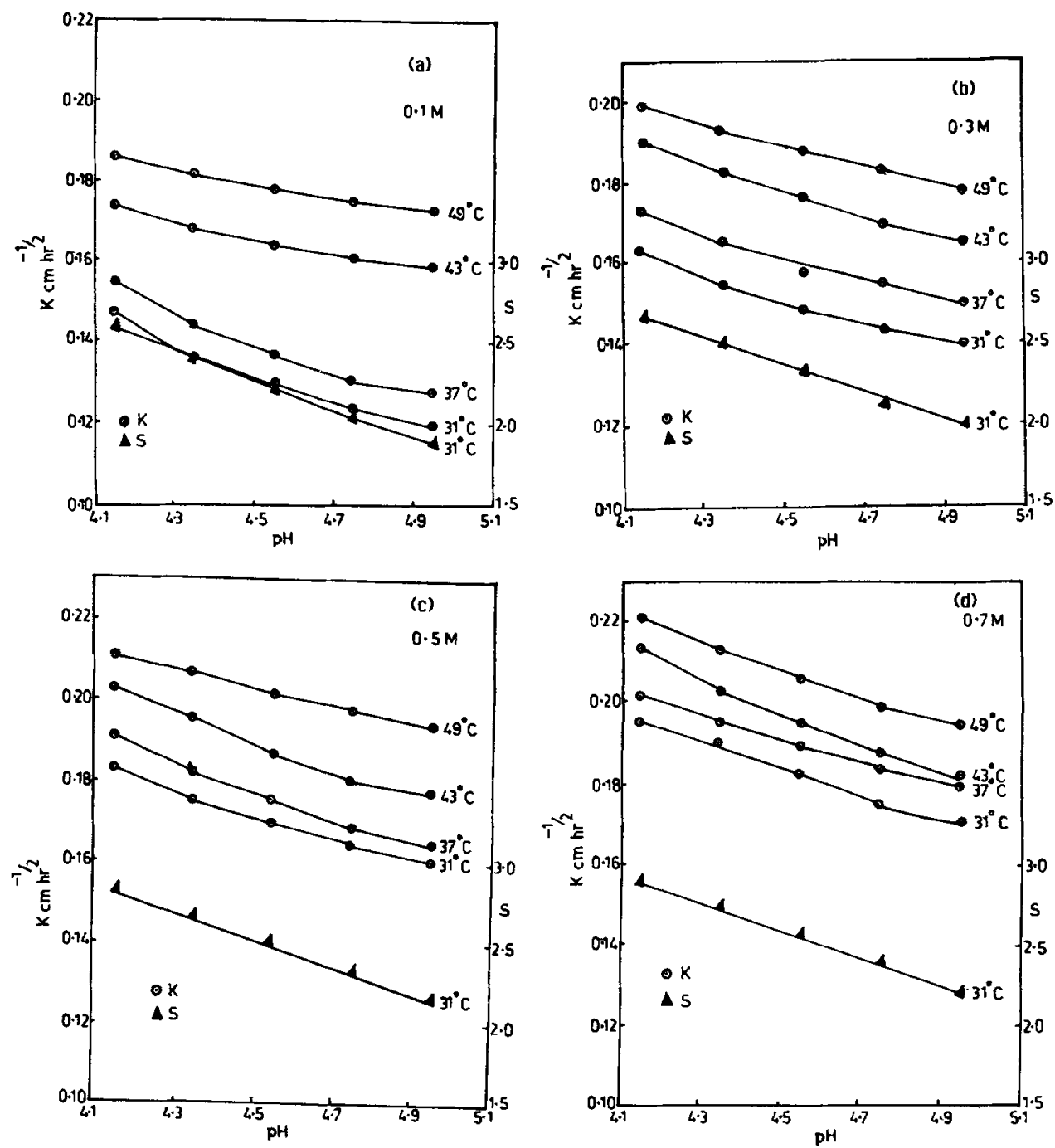

Figure 2. Plot of time constant $K$ and space constant $S$ against gel $\mathrm{pH}$ at various temperatures using (a) $0.1 \mathrm{M}$ (b) $0.3 \mathrm{M}$ (c) $0.5 \mathrm{M}$ (d) $0.7 \mathrm{M} \mathrm{BaCl}_{2}$ solutions.

With increased concentration, the diffusion rate of $\mathrm{Ba}^{2+}$ ions is enhanced which explains the greater value of velocity constant $K$. When a larger amount of outer electrolyte diffuses fast into the system containing uniformly distributed inner electrolyte, precipitation begins at the interface and the ring formation occurs below the interface, the growth of which is facihitated from the stoichiometric interior ions diffusing up to the position of the nucleated ring to provide nutrients to the growing crystals. This leaves a larger space below the ring which is depleted of inner electrolyte. Therefore, for the next ring to nucleate, the outer ions have to cross over this barren depletion region to meet with the other reactant. Since the passage is clear without any inner electrolyte, and $\mathrm{Ba}^{2+}$ ions diffuse more rapidly, it leads to increase in $K$. 

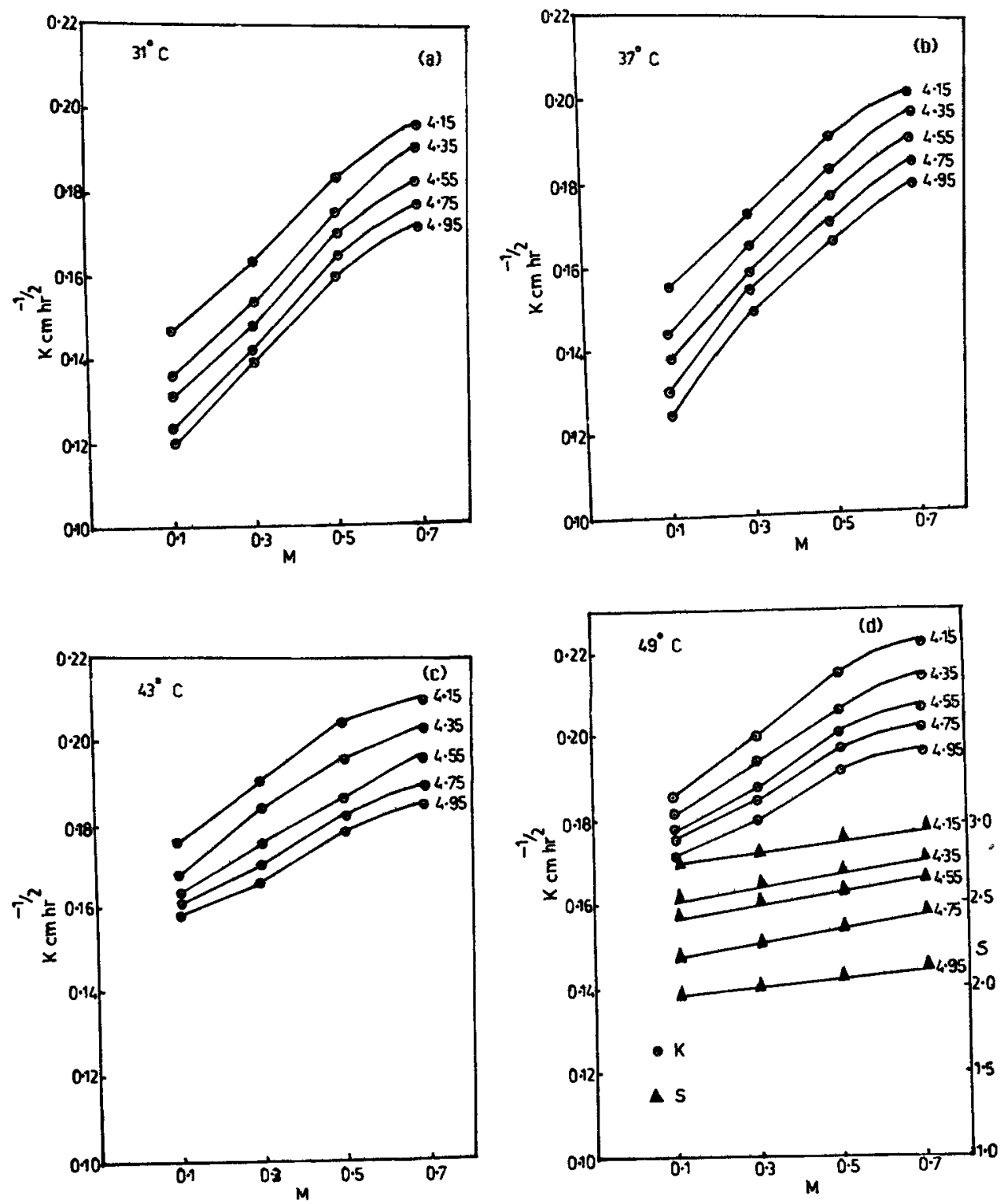

Figure 3. Plot of time constant $K$ against concentration of $\mathrm{BaCl}_{2}$ as outer electrolyte using different pH values at (a) $31^{\circ} \mathrm{C}$ (b) $37^{\circ} \mathrm{C}$ (c) $43^{\circ} \mathrm{C}$ (d) $49^{\circ} \mathrm{C}$ as well as space constant.

According to Isemura (1939), as the concentration of the outer electrolyte increases, the number of bands also increases and the successive interval between them decreases. Barium oxalate rings exhibit a different behaviour so that the total number of bands formed does not vary but the spacings between them increase noticeably with higher concentrations of barium chloride. The contradiction of our results when compared to Isemura's observation can be understood if we consider the nature of rings in both the cases. In amorphous or microcrystalline rings the 
constituting particles do not tend to grow up after the ring formation, whereas in crystalline rings the nucleated crystals do receive nutrients from larger distance because the growth of the already nucleated crystal is energetically favourable. This leads to greater separation between crystalline rings.

\subsection{Variation of $K$ and $S$ with temperature}

Figure 4 shows the plot of $K$ versus temperature and figure 5 the plot of $S$ versus temperature. The increased diffusion rate at higher temperature may be responsible for the increase in velocity constant. There is no appreciable change of space constant with change of temperature for a given $\mathrm{pH}$ and outer electrolyte
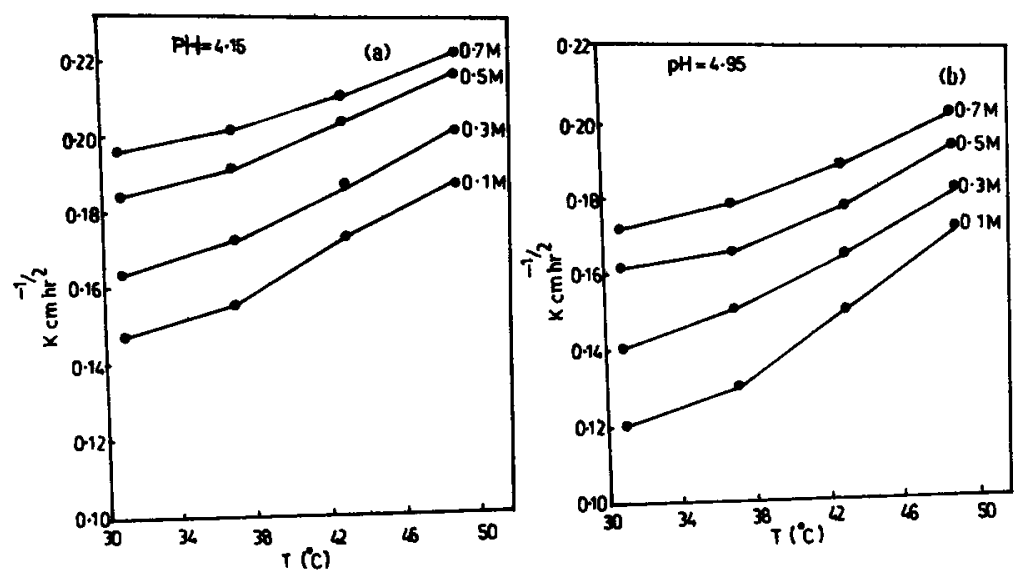

Figure 4. Plot of time constant $K$ against temperature using different concentration of the outer electrolyte at $\mathrm{pH}$ values of (a) $4 \cdot 15$ and (b) 4.95 .

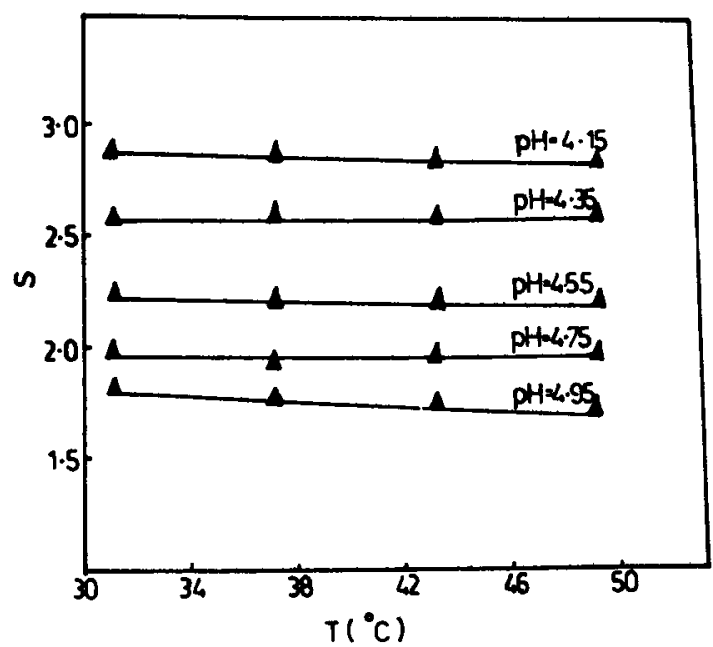

Figure 5. Plot of space constant $S$ against temperature at different $\mathrm{pH}$ values. 
concentration. This may be because of the competitive increase in the rate of diffusion of both the electrolytes.

Another interesting observation is that the barium chloride incorporated gel fed with oxalic acid as the supernatent solution produces no rings but only dense precipitation. This again contradicts Isemura's (1939) results according to which the interchanging of the reactants (as inner and outer electrolytes) will not affect the appearance of periodic precipitation. The nonappearance of rings when $\mathrm{BaCl}_{2}$ is impregnated with the gel is perhaps due to non-diffusion of the heavier barium ions in the upward direction with sufficient velocity required for the ring formation. The continuous diffusion of oxalate ions from the top results in continuous precipitation.

\section{References}

Arora S K, Tomy Abraham and Godebole 1982 Crystal Res. Technol. 17979

Bradford S C 1922 Kolloid $Z h .30364$

Dhar N R and Chatterji A C 1922 Kolloid $Z h .31151$

Gnanam F D, Krishnan S, Ramasamy P and Laddha G S 1979 J. Colloid Interface Sci. 73193

Hedges E 1932 Liesegang and other periodic structures (London: Chapman)

Isemura T 1939 Bull. Chem. Soc. Jpn 14179

Jablczynski K 1923 Bull. Chem. Soc. France 331592

Kannaiah N, Gnanam F D and Ramasamy P $1979 J$. Colloid Interface Sci. 80377

Liesegang R E 1896 Naturwiss Wochemschr 11353

Matalon R and Packter A 1955 J. Colloid Sci. 1046

Morce H W and Pierce G W. 1903 J. Phys. Chem. 45589

Ostwald W 1897 Lchrbuch der allgemeinen Chemie, Leipzig 788

Ostwald W 1925 Kolloid $Z h 36380$

Shinohara S 1970 J. Phys. Soc. Jpn 291073

Wagner C 1950 J. Colloid Sci. 585 